\title{
Low-Complexity Visible Light Networking with LED-to-LED Communication
}

\author{
Domenico Giustiniano, Nils Ole Tippenhauer, Stefan Mangold \\ Disney Research \\ Zürich, Switzerland
}

\begin{abstract}
Visible Light Communication (VLC) is an emerging technology in which Light Emitting Diodes (LEDs) transport information wirelessly, using the visible light spectrum. While most of the research on VLC has focused on wideband white LEDs used in ambient illumination, narrowband and colored LEDs have received little attention. Short-range free-space optical communication based on narrowband LEDs as visible light transmitters and receivers enable a variety of applications, a scenario we refer as LED-to-LED communication. In this paper, we introduce the communication and networking protocols of LED-to-LED communication. Our work addresses fundamental challenges such as efficient collision detection medium access protocol and elimination of light flicker. We build a prototype and demonstrate bi-directional data exchange in a network of up to four LEDs. We further study the trade-offs in the system design and measure the achievable bit-rate and transmission distances.
\end{abstract}

\section{INTRODUCTION}

Visible Light Communications (VLC) is an emerging alternative to traditional short-range wireless Radio Frequency (RF) communication [1]-[4]. Light Emitting Diodes (LEDs) have become the technology of choice because of their reliability and low cost. The brightness of LEDs can be adapted rapidly, at speeds orders of magnitude higher than for conventional light emitting devices. By varying the intensity of the LED light source, data messages can be communicated through visible light to a receiver sensitive to light. LEDs emit light but can also be used as receivers to measure incoming light, just like photodiodes, which permits to transmit in point-to-point links [1]. We refer to this approach as $L E D$ to-LED communication. In LED-to-LED communication, we can control the communication through the directivity and the visual field of light propagation, which makes it interactive (in contrast to infrared or RF) [5].

In this work, we address fundamental challenges to use LEDs for bi-directional communication and build a complete LED-to-LED network. A fundamental characteristic is that the visible light signal sent by an LED can, at the same time, be seen as a light source, whereas the intended receiver interprets it as source of information. Therefore, communication between optical devices can lead to experiences of undesirable flicker for nearby human observers. In addition, a communication protocol that coordinates the access to the optical wireless channel is needed to realize a communication network. Adhoc protocols should be selected such that the system scales towards larger number of devices.
This paper's contributions are as follows. After related work section (Section II), we present a complete LED-toLED network system in Section III. Our target is to build a low-complex network system where each device is composed by one narrowband LED and one microcontroller. Using a bottom-up approach, we start with the basic circuit used for LED-to-LED communication, following the method of [1]. Based on this design, we introduce a flickering elimination method and use LEDs to measure incoming light not only during receiving messages (as part of receiving data), but also during transmission. This concept allows us to introduce a carrier-sense protocol based on free-space optical collision detection to limit the impact of network collisions.

In Section IV, we study the MAC throughput in an experimental optical network of up to four LEDs. Next, we show through measurements that LED sensitivity, physical rate and flicker elimination are tightly correlated, which calls for trade-offs in the system design. We address and evaluate design parameters with several tests and analytical insights. We further study the impact of noise, such as the LED current and the ambient light. In Section V, we finally give details on our prototype implementation, and we demonstrate LED-to-LED communication showing time series of data/ACK exchange. Conclusions are drawn in Section VI.

\section{RELATED WORK}

The pioneering work of the Japanese VLC consortium [6] has led to widespread interest within the research community. The Visible Light Communications Consortium (VLCC) was established in November, 2003, with major companies in Japan and aims to publicize and standardize the VLC technology. The recent IEEE 802.15.7 standard [4] exploits LEDs for wireless data transmission in Wireless Personal Area Networks (WPAN). The standard uses wideband white LED used for illumination for high-speed communication. Towards high PHY rate, the Omega project [7] achieved $100 \mathrm{Mb} / \mathrm{s}$ using LEDs on ceiling lighting. In contrast, we target narrowband $(15-150 \mathrm{~nm})$ LEDs in low-complexity applications and with low PHY rate, as these are sufficient for cost-effective applications in entertainment, such as toys.

Another main difference is that the primary goal of wideband white LEDs in 802.15.7 and Omega project is illumination, while our primary goal is communication and colors are used as visual emotion/feedback. The consequence is that our target applications can be used at any time, even 
when no white LED illumination is required. Our goal is to investigate the limit of communication using a single LED and microcontroller for applications that require low PHY rate. Using low-cost LEDs not only as light emitters but also as light detectors (receivers) reduces the amount of components. No other optical or electronic components are required by our design. While resistances, operation amplifiers, etc. can increase the communication rate and range, they also increase power consumption and cost per-device. Besides, using LEDs as receivers offer other interesting advantages over using photodiodes (PDs). A normal LED's sensitivity region is only slightly wider than its spectral emission profile, while PDs indiscriminately detect a wide spectrum of visible and infrared light. Therefore, no additional optical filters are needed for LEDs [8], [9], which makes LEDs more robust against interference from sunlight and against any source of man-made interference.

The IEEE 802.15.7 presented a slotted random medium access protocol similar to what we use in our implementation. However, the MAC protocol expects normal photodiodes as receivers. Our protocol includes a collision detection mechanism, taking advantage of the sensing capability of LEDs, and compatible with our flicker elimination scheme. Flicker problems are well studied in fields like visual displays [10], lamps [11], and video games [12], but only partially addressed in current VLC designs. The 802.15.7 standard proposes to always keep the channel busy by transmitting messages with dummy data content. To avoid intra-message flicker, the standard proposes to use Manchester or 4B6B coding [4]. The 802.15.7 standard addresses the flickering problem only for the master device in infrastructure mode, but not in the other devices of the network. When in ad-hoc mode, it permits the devices to flicker. In contrast, our solution globally resolves the flickering problem for each station of the entire network.

The InfraRed Data Association (IRDA) has developed protocol stacks for point to point links in Personal Area Networks (PAN). IrDA was popular on PDAs, laptops and some desktops during the last decade of 20th century. The latest GigaIR standard uses lasers, rather than LEDs, which makes it more expensive [13]. Besides, due to the absence of visible light feedback, the system design space of our LED-to-LED communication differs from infrared communication, and most of the advantages of VLC and its interactive applications would disappear in infrared communication.

\section{LED-TO-LED COMMUNICATION}

Use cases and applications of LED-to-LED communication are characterized by low-cost and low-complexity (toys, consumer electronics). This requires low PHY data rates for exchanging messages and typically a simple ad-hoc nature of the communication network established among visible light emitters and receivers. These requirements must be fulfilled by a lightweight architecture and communication protocol and a simple transceiver circuit based on one LED and one microcontroller as described next.

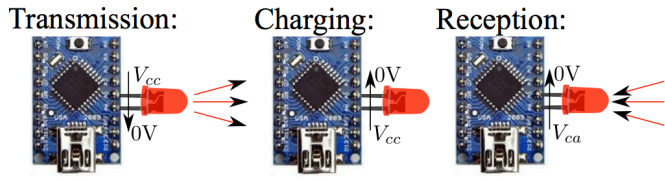

Fig. 1. LED operating modes for transmission and reception. The LED anode is connected to a digital pin and the cathode to an ADC pin.

\section{A. Communicating with an LED}

A simple and low-cost incoherent optical modulation and demodulation can be realized via intensity modulation (IM) at the transmitter with direct detection (DD) at the receiver [2]. At the transmitter, we use an on-off keying (OOK) [14], where binary information is mapped to the presence (symbol ONE) or absence (symbol ZERO) of the optical signal. In our design, the LED is used both as transmitter and receiver front-end, and hence, is capable of bi-directional communication. Our design only requires minimal components, as the LED is directly connected to a digital IO pin and an Analog to Digital (ADC) input / digital output pin (see Fig. 1). The digital IO pin is connected to the anode of the LED, and the ADC pin is connected to the cathode. Next, we describe why this setup enables us to both transmit and receive visible light signals.

In our system, a ONE symbol is transmitted by emitting light for time $T$ and a ZERO is transmitted by emitting no light for time $T$. The emitted light causes a small reverse current in any receiving LED. The light sensitivity of LEDs is low compared to photodiodes, because of the smaller reception area and less collected light, and, hence, it cannot be directly measured by a standard ADC pin of a microcontroller. Instead, we use the principle originally presented in [1] to detect light with an LED: we use the reverse current generated by the incoming light to slowly discharge a capacitor, that corresponds to the integration of the incoming light during the period of measurement ${ }^{1}$. We will now describe transmission and reception in more detail.

1) Symbol ONE: The LED is supplied in forward bias at a voltage $V_{c c}$ when transmitting symbol ONE, such that current flows from the anode to the cathode. The intensity of the light emitted by the LED is proportional to the current flowing between the anode and the cathode. Typically, microcontrollers provide limited current on their pins and we do not require an additional current limiting resistor for the LED.

2) Symbol ZERO and Reception: To transmit a ZERO symbol, it is sufficient to supply the LED below the threshold $V_{\gamma}>0$, which guarantees absence of light emission. We use the following key idea: Rather than driving the LED at no bias (as usually done in optical communications), to sense the channel, we operate the LED in reverse bias when we transmit

\footnotetext{
${ }^{1}$ [1] used two digital I/O pins rather than a digital I/O pin and an ADC pin as in this work. As a result, Dietz et al. needed to measure the time needed for the charged LED to decay down to a predefined threshold. The absence of an ADC pin does not allow either a dynamic threshold per-message or comparison of energy measured in consecutive symbols, necessary to both optimize the range of communication (of only $3 \mathrm{~cm}$ in [1]) and ensure that a global synchronization is possible in a LED-to-LED network. Furthermore, [1] works only with point to point links, and it does not consider features as measuring the energy at the time of transmitting symbol ZERO.
} 


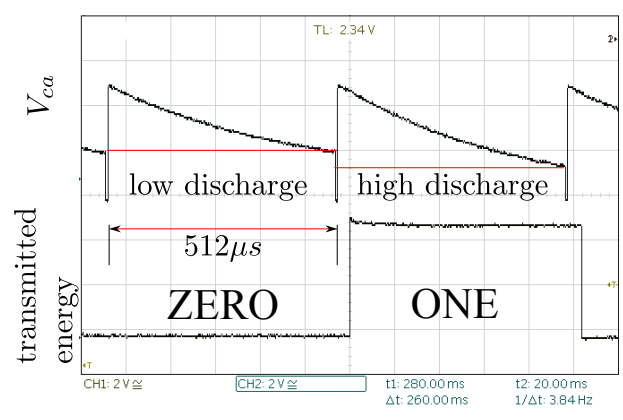

Fig. 2. LED capacitance discharge due to received light using a symbol period of $T=512 \mu \mathrm{s}$. At the bottom we show the signal transmitted and at the top the one received. The discharge is slower when it receives ZERO.

a ZERO symbol. The advantage is that light can be sensed when ZERO is transmitted as explained next.

The incoming light is measured as follows. First, a capacitor with capacitance $C$ is charged to some reference voltage applying a reverse bias. Due to the high output current of the microcontroller (compared to the capacitance $C$ ), this charging requires only few nanoseconds. Once the capacitance is loaded, it is slowly discharged by the LED in reverse bias mode. At the end of some period, the remaining charge of the capacitor is measured by the microcontroller. Fig. 2 gives an example of the discharging process during the data reception in our prototype setup. At the bottom we show the signal transmitted, and at the top the one received. The transmitter sends ZERO and a subsequent ONE. The receiver measures the voltage at the end of the symbol period. This discharge decay is shown in the top graph of Fig. 2. The discharge is quicker when it receives ONE due to incoming light which generates a photocurrent $I_{\text {photo }}$ in the $\mathrm{LED}^{2}$. In practice, the internal capacitance of an LED can be used for this, without the need of additional components. Let us denote $V_{c a} \geq 0$ the voltage between the LED (cathode and anode) pins. The symbol decision is made after i) charging the internal LED capacitance $C$ in reverse bias to $V_{c a}=V_{c c}$ and ii) measuring the voltage drop after a symbol period $T$. Comparing $V_{c a}$ to a threshold $V_{t h}$, the decision at the receiving LED is:

$$
\begin{gathered}
V_{c a}(T)>V_{t h} \rightarrow \text { symbol }=\text { ZERO } \\
V_{c a}(T)<V_{t h} \rightarrow \text { symbol }=\text { ONE. }
\end{gathered}
$$

\section{B. Flicker Elimination}

In some aspects, the communication in visible light largely differs from radio communication. We partition the frequency domain into several regions from the perspective of a human observer, as described in the following:

Visual feedback region: The low-pass component of the visible light signal is the one observed by human eyes. The human eye has also provisions to tolerate a considerable dynamic range in the light illumination by adjusting the aperture of the iris. Thus, small variations of the light intensity

${ }^{2}$ In Fig. 2, $C$ is discharging faster than normal for both ONE and ZERO due to the connected oscilloscope probe. In normal operation, the charge decay is less steep for reception of ONE and ZERO symbols can be tolerated. This is also the portion of spectrum that can be used to perceive establishment of connection and range of communication. The signal contained in this spectrum not necessarily conveys information to the remote LED station.

Flicker region: Flickering is observed when the variation of illumination of a light source is noticeable to the human eye [10], [15]. An important factor is the flicker's frequency, which can reach hundred Hertz. The effect of flicker can be even more noticeable in a network where different transmitters are lit up at different time to transmit their messages. Hence, there exists an intermediate region in the frequency domain in which our signal should have low or no energy at all. Signals in this zone are caused by long runs of only ONE (or ZERO) symbols during the transmission of a message (denoted as intra-message flickering). Flicker can also occur during periods of inactivity during message transmission, or while messages are received (denoted as inter-message flickering).

LED-to-LED data region: the region at higher frequencies can be used for optical communication. Human eyes can not detect these signals, as the temporal response of the visual system filters high frequencies, attributable to limitations in the rate at which the nervous system can respond [16].

1) Design of flicker elimination: The resulting optical signal has to be composed by a low-pass and high-pass signal component only, in order to avoid the prohibited flicker region. A simple solution to mitigate both sources of flicker would be to increase the symbol rate $1 / T$, which will not be perceived by the human eye any more. However, this is not a valid option, since the increase of $1 / T$ would reduce the symbol period $T$, and thus the sensitivity range of an LED. Other options as Manchester coding or similar line coding are also not sufficient for our scope, since they can only provide intra-message flicker compensation (after synchronizing to the message), but do not provide any solution to inter-message flicker. A scheme to globally eliminate the effects of both sources of flicker is introduced in the following.

We assume a network setting with two or more stations sharing a common optical wireless channel, e.g. in the red or yellow spectrum. Each LED station $i$ emits in average a level of energy $\bar{E}_{i}$. We divide the communication channel into symbols of time duration equal to the symbol period $T$ and introduce the concept of Data and Energy symbols. The device transmitting a message accesses the channel and sends the frame using the Data symbols, according to the data communication protocol introduced above.

2) Data Symbol: The Data symbols are used to i) either receive any message from stations in range or listen the channel before transmitting any message ii) carry any information (bit) from one transmitter to all receivers in range. In the former case, the station sends symbol ZERO. In the latter, station transmits the bits using the binary OOK modulation as follows:

$$
\begin{aligned}
& \text { bit }=0 \rightarrow \text { symbol }=\text { ZERO } \\
& \text { bit }=1 \rightarrow \text { symbol }=\text { ONE. }
\end{aligned}
$$

The Data symbols are exclusive, i.e. only one transmitter is allowed to transmit signals in each Data symbol. All 

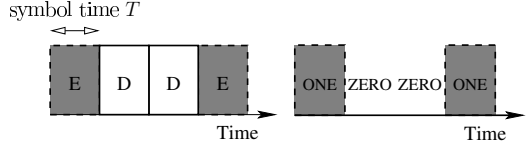

(b) Reception.

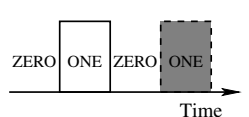

(c) Transmitter, sending data bit ' 10 '.

Fig. 3. Flicker Elimination Scheme: (a) The channel is partitioned into Data (D) and Energy (E) symbols. (b) The receiver emits light only during the Energy symbols. The same pattern is sent when the channel is idle. (c) The transmitter emits light during the Data (for the message) and eventually, during the Energy symbol (to compensate the flicker).

medium access and higher layer communication protocols (Section III-C) are relying on frames exchanged during the Data symbol. During the transmission of a message, no station in communication range (other than the current transmitter) should transmit ONEs during the Data symbol.

3) Energy Symbol: In the Energy symbols, no data is transmitted by any stations. During the Energy symbols, any transmitter is free to output energy to the optical medium. All stations can choose to use the Energy symbols to output signals in order to maintain a specific average power output level $\bar{E}_{i}$ of its choice. For example, every ZERO transmitted in a Data symbol can be compensated by a ONE in an Energy symbol, and vice versa. The pulses in the Energy symbols do not carry any information and are not intended to be received by other stations, but rather by the human eyes. They may also use different intensity, if needed and supported by the transmitter. This way, a transmitter can maintain the constant target power output level throughout the transmission of a message and hence any flicker of the emitted light will be eliminated.

4) Flicker encoding scheme: When a station in the network is active, it starts to emit an average light $\bar{E}_{i}$, corresponding to a sequence of Data (D) and Energy (E) symbols equal to EDDE, as shown in Fig. 3(a). The first Energy symbol is used to compensate the first (following) Data symbol, and the second Energy symbol compensates the preceding second Data symbol. The default state of each station is the reception, where it listens to the channel. As shown in Fig. 3(b), the receiver sends symbol ZERO when it may expect to receive any data from other stations. For anti-flickering elimination while in reception, every station sends ONEs during the Energy symbols. The resulting sequence is [ONE ZERO ZERO ONE]. A station with a message ready for transmission and RBT equal to zero can emit light during the Data symbols. In the example in Fig. 3(c), a transmitter sends bit 1 (symbol ONE) followed by bit 0 (symbol ZERO), which are compensated by a symbol ZERO preceding the first Data symbol and a symbol ONE following the second Data symbol. As a consequence, an observer will not notice any differences in light emission for transmission and reception, switching between the two phases, or between listening to the channel or transmitting.

\section{Medium Access and Networking Protocol}

To ensure a fair access to the medium for all stations, we introduce a contention-based MAC protocol based on collision detection, and compliant with the flicker elimination scheme

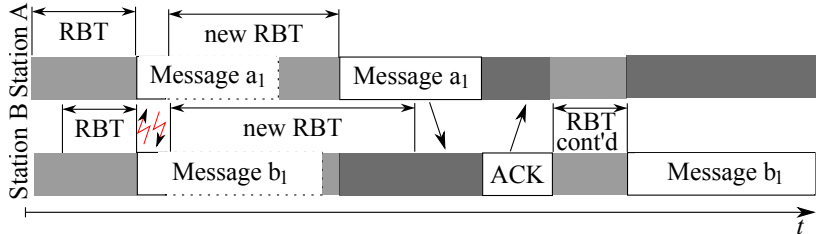

Fig. 4. MAC-layer Message retransmission mechanism with CSMA/CD.

above introduced. We first consider an example of access to the channel when there is only one transmitter (station A) and one receiver (station B). A Random Backoff Time (RBT) is generated before transmitting a message. The RBT is an integer value selected from an uniform distribution with range $\left[0, \mathrm{RBT}_{\min }-1\right]$. While a transmitter's counter is counting down, the transmitter senses the optical medium to detect message transmission attempts by other stations on the network. Station A decrements RBT by one unit after no activity (idle channel) is detected for a time equal to the MAC_SlotTime. While station A is counting down, it measures the activity of the channel only in the fraction of symbols dedicated to Data symbols (where it sends ZERO symbols). Energy symbols cannot be used since the transmitter sends ONE symbols for flicker compensation. The data message is transmitted after the timer reaches zero. ACK message is transmitted by station B immediately after reception of the message ${ }^{3}$. Once the ACK is received, station A generates a new RBT in the range $\left[0, \mathrm{RBT}_{\min }-1\right]$ for next message. Each time the ACK is not received within a time ACKTimeout, the message is retransmitted again after a new RBT, where RBT is doubled to reduce the risk of future collisions. A maximal value $\mathrm{RBT}_{\text {limit }}$ limits RBT, so that this variable cannot grow unlimited. After a maximum number of attempts, the message is dropped

1) Collision Detection (CSMA/CD): If two or more LED stations transmit frames at the same time, a collision will occur. If the sender requested an ACK, it can infer that a collision happened when the ACKs are not received. Since we target low PHY rate communication, the time wasted in the collision can cause an inefficient utilization of the optical channel, even for small number of contending stations. In the following, we introduce a solution to this problem which takes advantage of the sensing capability of LEDs during transmissions of ZERO symbols.

We refer to Fig. 4. When a LED station gains access to the optical medium and transmits the message, it expects that no light is emitted by other LED stations during the Data symbols. While the LED cannot sense the medium when it transmits a ONE during the Data symbol, it can do so while transmitting a ZERO during a Data symbol to transmit a bit 0 of the message. The transmitter uses the technique presented in Section III-A2 to measure the activity on the channel. The transmitter expects

${ }^{3}$ The ACK message uses a MAC layer format without data content (i.e., data payload equal to zero byte), simply acknowledging the successful reception. To reduce the overhead of the communication, ACKs are sent with shorter sync sequence equal to one preamble since the data transmitter and receiver are already synchronized. Since LEDs can be switched from forward to reverse bias in a time in the order of one $\mu \mathrm{s}(\ll T)$, there is no turnaround time to wait before sending the ACK. 
that no activity is detected during its transmission. Whenever another LED station starts to transmit its message concurrently, each of the stations colliding will detect the presence of activity on the channel during the transmission of bit 0 and stop to transmit immediately when energy above a threshold is detected. This method enables a carrier sense multiple access with collision detection (CSMA/CD) protocol. To increase the robustness, a decision about the presence of channel activity is taken after each MAC_SlotTime.

\section{LED-TO-LED SYSTEM TRADE-OFFS}

In this section we investigate what parameters limit the performance of LED-to-LED communication. In particular, the choice of the symbol period $T$ is driven by i) flickering of light, ii) the noise generated by the LED in reverse bias, and iii) the target communication rate and range. We find that:

- Using payload of up to 160 byte, the maximum system throughput $S_{\max }$ on one color is $S_{\max } \approx 0.45 / T$.

- The maximum symbol period is given by $\min \left\{T_{Z E R O}, \frac{1}{4 C F F}\right\}$, where $T_{Z E R O}$ is the time where the capacitance starts to discharge due to noise sources, and CFF is the Critical Flicker Frequency, frequency at which flicker starts to be observed.

- The voltage $V_{c a}$ measured at the end of the symbol period depends on the communication distance $d$ as $\left(V_{c c}-V_{c a}(T)\right) \propto 1 / d^{2}$.

\section{A. VLC System Prototype Implementation}

Our visible light transceiver is composed by a digital and analog part. The digital part consists of data source, a driving circuit controlled by an Arduino microcontroller $(\mu \mathrm{C})$ with built-in analog-to-digital converter (ADC) [17]. The analog front-end is an LED. We supply the LED at $V_{c c}=5 \mathrm{~V}$, without damaging the LEDs, as the output current is limited to $20 \mathrm{~mA}$. Unless otherwise specified, each transceiver uses a symbol period of $T=512 \mu \mathrm{s}$. Symbol boundaries are obtained via interrupts generated by the Arduino and handled by our firmware. The resulting hex file is of about $11 \mathrm{kB}$.

\section{B. Achievable Throughput}

We measure the achievable throughput as the number of MAC data bits successfully acknowledged in average in a second using our prototype implementation. We run tests up to four transmitters sending data on the red spectrum with payload of 40 and 160 byte, with collision detection (CSMA/CD) and without (CSMA/CA), for a symbol duration of $512 \mu \mathrm{s}$. Each test lasts for $600 \mathrm{~s}$. We summarize the results in Fig. 5. We measure a system throughput of up to 872 bit per second (b/s) for messages of 160 byte. The throughput decreases for shorter message length. We obtain a Jain's fairness index [18] of $0.98-1$, which indicates that both our protocol and prototype implementation are fair. As expected, with CSMA/CA, the system throughput decreases while increasing the number of nodes, due to the channel contention and collisions. Enabling the CSMA/CD, the system

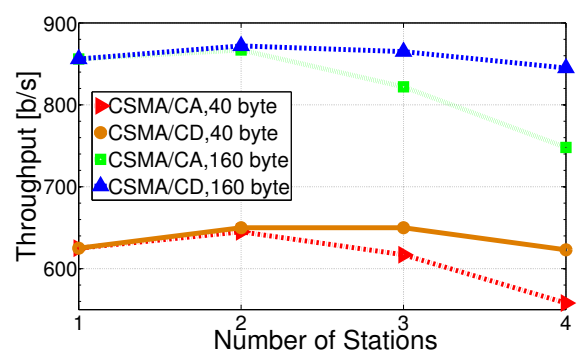

Fig. 5. MAC throughput (in bit per second, b/s) versus the number of LED transceivers. The legend shows the length of data payload per message. The throughput indicated is the total system throughput. Collision detection is indicated as CD. In the tests, we obtain a Jain's fairness index of $0.98-1$, that indicates that both our protocol and prototype implementation are fair.

throughput can obtain higher throughput when multiple transmitters are contending for the optical medium, thanks to the early detection of a collision. Furthermore, the throughput gain with $\mathrm{CD}$ is higher with longer data payload, as a consequence of the longer temporal size of the messages and the time saved with an early detection of collision events.

1) Theoretical Maximum System Throughput: The maximum system throughput obtained with CSMA/CD is very close to the one expected from theory. Indeed, given the probability $P_{s}$ to successfully access the channel and the average payload bits $E[P]$ of the transmitting LED, the system throughput $S$ can be expressed as $S=\frac{P_{s} E[P]}{E[\text { slot }]}$, where $E[$ slot $]$ is given by the sum of three components: 1) the channel inactivity due to backoff count-down. 2) the channel occupancy time of the message due to successful transmission $P_{s} \cdot E\left[T_{m}\right]$ 3) the channel occupancy time due to collision, equal to $\left(1-P_{s}\right) \cdot E\left[T_{c}\right]$, where $E\left[T_{c}\right]$ is the average time wasted in a collision [19]. Due to our low symbol-rate, message transmission can take relatively long time. For example, a message with payload $P=160 \cdot 8$ bit, ACK (no payload, see Sec. III-C) and their PHY preambles occupy the channel for $(352 \cdot 8) \cdot T=1.44 \mathrm{~s}$. For small number of collisions and using collision detection, this time is much longer than the time spent in the backoff count-down and in a collision. As a result $E[$ slot $] \approx P_{s} E\left[T_{m}\right]$ and the maximum system throughput $S_{\max }$ in CSMA/CD is:

$$
S_{\text {max }} \approx \frac{E[P]}{E\left[T_{m}\right]}=\frac{160}{352 \cdot T}=0.45 / T \text {. }
$$

For $T=512 \mu \mathrm{s}, S_{\max }=878 \mathrm{~b} / \mathrm{s}$, which is very close to what we obtain in our tests in Fig. 5. Eq. (1) permits also to calculate $S_{\max }$ on one color varying $T$. For example, a system throughput of up to $1.77 \mathrm{~kb} / \mathrm{s}$ is expected with $T=256 \mu \mathrm{s}$, and $S_{\max }=444 \mathrm{~b} / \mathrm{s}$ with $T=1024 \mu \mathrm{s}$.

\section{Flicker Mitigation due to Run Length Limiting}

As discussed in Section III-B, we have to ensure that the visible light signal does not vary in intensity at frequencies leading to perceived flicker for nearby humans. Therefore, we have to ensure that our choice of $T$ will not lead to unwanted frequencies in the signal. Based on research on human flicker sensitivity [16], [20], the maximum luminance and the 
minimum refresh frequency observed by a human viewer can be predicted simply based on the lowest frequency of the baseband signal, called the Critical Flicker Frequency (CFF). When the signal varies at frequencies higher than the CFF, the observer does not perceive flicker. It follow that we only need to know the amplitude of the CFF, regardless of the higher signal components and the ambient illumination. From [20], the solution to the flickering problem is to guarantee that the absolute amplitude $E$ at frequency $f_{\min }$ is greater than $E_{F}\left(f_{\text {min }}\right) \propto \exp \left(a 2 \pi f_{\text {min }}\right)^{1 / 2}$, where $a$ is a constant. This translates to guarantee an energy $E_{F}\left(f_{\min }\right)$ sufficiently small, or, because of the exponential relation between $E$ and $f_{m i n}$, increases $f_{\min }$ so that higher energies are allowed. Without any flickering elimination scheme, $f_{\min } \ll 1 / T$, especially due to inter-message flicker. As shown in Fig. 3, our antiflickering encoder limits the length of same symbol runs to two symbols (thus, $2 \cdot T$ ). This signal can be represented as a square wave with period equal to $4 T$ and the lowest frequencies in its spectrum are at $f_{\min }=1 /(4 T)$, much higher than without this run length limiting.

1) Experimental analysis of flicker: We verify the above analysis in our implementation. We collect signal traces of the transmitted signal with and without inter-message flicker elimination $(6000$ samples, at $10 \mathrm{KHz})$. In the test, the LED transmits data messages to another LED. Results are shown in Figure 6. On the left, we show an example without eliminating flicker. As expected, there are low frequency components (below $100 \mathrm{~Hz}$ ) that can cause flicker effects to humans and $f_{\min } \ll 1 / T$. On the right, we illustrate the case of flicker elimination. The spectrum is such that the average light emitted $(0 \mathrm{~Hz})$ is visible to human and average higher than absence of flicker compensation, but higher visible frequency components are attenuated by up to $20-25 \mathrm{~dB}$. The signal has a stronger peak at $\approx 500 \mathrm{~Hz}$, as expected from $f_{\min }=1 /(4 T)$, which can not be seen by human eyes. The result is a strong reduction of flicker.

We also evaluate for which symbol period $T$ we start to visually observe flicker even when using flicker compensation We vary $T$ and transmit data messages using our flicker elimination mechanism to another node in range. In our experiments, we start to observe a slight flicker with $T=1.5 \mathrm{~ms}$, which results in $f_{\min }=C F F=\frac{1}{4 T}=166 \mathrm{~Hz}$. Note that different results may be observed for different output LED currents (20 mA of our tests) (see Section III-B).

\section{Noise from LED Current and Indoor Illumination}

At the receiver, the symbol period $T$ is limited by the dark current generated in the semiconductor junction when supplied in reverse bias. This current is a source of noise, since it discharges the capacitance $C$ both in absence of communication and when receiving symbol ZERO. A second source of noise is the pollution generated by the ambient illumination, as the one generated on the optical spectrum by modern lighting devices for indoor illumination. Because of the finite resolution to represent the voltage in the microcontroller, the sensitivity of the voltage between the cathode and the anode is limited. It
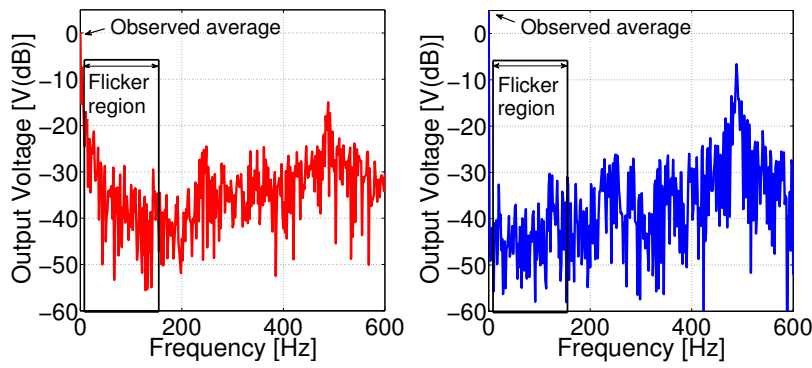

Fig. 6. Frequency analysis of signal $(6000$ samples at $10 \mathrm{KHz})$. On the left we show an example where there is no flicker compensation between messages. There are low frequency components (below $100 \mathrm{~Hz}$ ) that can cause flicker effects to humans. On the right, we show the spectrum with our flicker elimination scheme. The spectrum is such that the average light emitted $(0 \mathrm{~Hz})$ is visible to human and is used as visual feedback, but higher visible frequency components are attenuated by up to $20-25 \mathrm{~dB}$. The signal has a stronger peak at $\approx 500 \mathrm{~Hz}$, which can not be seen by human eyes.

follows that the voltage $V_{c a}$ measured with the ADC does not change until a symbol period $T_{Z E R O} . T=T_{Z E R O}$ guarantees the maximum sensitivity since the LED collects the highest amount of light before making a decision about the presence of ONE or ZERO on the optical medium.

1) Experimental impact of noise: We evaluate the practical impact of the noise on our prototype. Results are summarized in Fig. 7, which displays $T_{Z E R O}$ in darkness (above) and indoor ambient light (below). We select $T_{Z E R O}$ as the value where fewer than $1 \%$ of measurements result in a voltage drop of the capacitance using a 10 bit ADC pin. In darkness, we are interested in the dark current, and we minimize the effect of any incoming light measuring in a dark environment, with the sensitive area covered with a black cardboard box. Under indoor ambient light, we characterize the indoor irradiance using TAOS TSL230rd photometric sensors installed on LabJack U3 DAQ device [21]. We measure an irradiance of $54 \mu \mathrm{W} / \mathrm{cm}^{2}$ in proximity to the LED under test. We use $3 \mathrm{~mm}$ and $5 \mathrm{~mm}$ LEDs $^{4}$ of color blue (B), yellow (Y), green (G) and Red (R). For example the label Y5 indicates a yellow $5 \mathrm{~mm}$ LED. In the tests we fix the symbol period in a scale multiple of $64 \mu \mathrm{s}$. From Fig. 7, we find that the ambient light impacts the discharge of the capacitance, especially on green and yellow LEDs. In fact, these LEDs are more sensitive to the green and yellow component of the visible spectrum [8], [9], where higher indoor ambient light noise is expected. For practical usages, blue and red LEDs are preferable since they are more robust to indoor ambient noise.

In Section IV-C we have that $T<1.5 \mathrm{~ms}$ prevents flicker using the design presented in Section III-B1. Referring to Fig. 7, we can infer than, in darkness conditions, the flicker frequency $C F F=\frac{1}{4 T}$ introduces an upper-bound to the maximum symbol period. The consequence is that we are forced to operate at smaller sensitivity that the maximum one achievable with $T_{Z E R O}$. Under ambient light, this noise is predominant in yellow and green LEDs, as well as in the $5 \mathrm{~mm}$

${ }^{4}$ Our LEDs are: BL-BB53V4V blue $5 \mathrm{~mm}$, BL-B3134 yellow $5 \mathrm{~mm}$, BLB2134 green $5 \mathrm{~mm}$, BL-B4534 red $5 \mathrm{~mm}$, BL-BB53V1 blue $3 \mathrm{~mm}$, BL-B3141, yellow $3 \mathrm{~mm}$, BL-B2141 green $3 \mathrm{~mm}$, BL-B4541 red $3 \mathrm{~mm}$. 

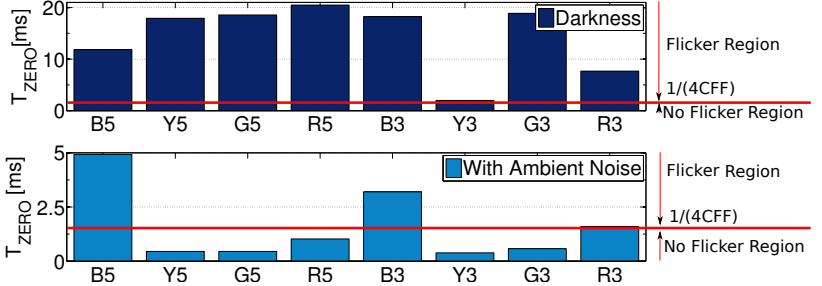

Fig. 7. Symbol period $T_{Z E R O}$ that maximizes the sensitivity for different 3 and $5 \mathrm{~mm}$ LEDs in darkness (above) and indoor ambient light (below) that irradiates at $54 \mu \mathrm{W} / \mathrm{cm}^{2}$. In darkness, the noise is due to the dark current. The ambient light impacts the discharge of the capacitance, especially on green (G) and yellow (Y) LEDs. In fact, these LEDs operate are more sensitive to the green and yellow component of the visible spectrum of the visible spectrum, where higher indoor ambient light illumination is present.

red LED, while flicker is predominant in blue LEDs and $3 \mathrm{~mm}$ RED. In next section we evaluate the practical impact of this result, measuring the achievable communication range with $T=\{256,512,1024\} \mu$ s where no flicker is present in all the blue and red LEDs under test.

\section{E. Communication Range}

We experimentally study the achievable distance using $5 \mathrm{~mm}$ LEDs with blue-to-blue and red-to-red communication. In the test, we have a blue (red) transmitter supplied at $V_{c c}=5 \mathrm{~V}$ that continuously emits light and a blue (red) receiver that measures the voltage drop after a symbol period $T$. LEDs are aligned to study the maximum range. In each measurement, we fix the symbol period $T=\{256,512,1024\} \mu$ s and the distance between the LEDs, and measure the voltage $V_{c a}$ after a symbol period $T$, according to the method presented in Section III-A2. We collect 1000 samples (symbols), we calculate the average voltage and plot it in Fig. 8 as a function of LEDs distance.

The plot shows that the communication range is a function of $T$ and the LED itself. Distances of $90 \mathrm{~cm}$ and more can be obtained using blue LEDs and $T=[512,1024] \mu \mathrm{s}$, while red LEDs can communicate only until $\approx 30 \mathrm{~cm}$. These distances may be sufficient in entertaining and interactive toys which will be likely located in a single room. As expected, a longer symbol period helps to collect more light and increases the communication range. For example, the blue LED measures an average voltage of $3.5 \mathrm{~V}$ at $15 \mathrm{~cm}$, when using $T=1024 \mu \mathrm{s}$ and an average value of $4.15 \mathrm{~V}$ using $T=512 \mu \mathrm{s}$. The results also demonstrate that the symbol period cannot be reduced arbitrarily (which would reduce any flicker effect without using our flicker elimination scheme) since smaller symbol periods reduce the range of communication.

Results in Fig. 8 follows an inverse square law. This can be explained considering that the intensity $I$ of light falling at a distance $d$ in a particular direction as $I \propto 1 / d^{2}$. As in every photodetector, the generated photocurrent $I_{\text {photo }}$ is proportional to $I, I_{\text {photo }} \propto 1 / d^{2}$. Since $I_{\text {photo }}$ is constant during the symbol time $T$, the same model can be applied to the measured voltage $V_{c a}(T)$, that integrates $I_{\text {photo }}$ over $T$. We conclude that $\left(V_{c c}-V_{c a}(T)\right) \propto 1 / d^{2}$.

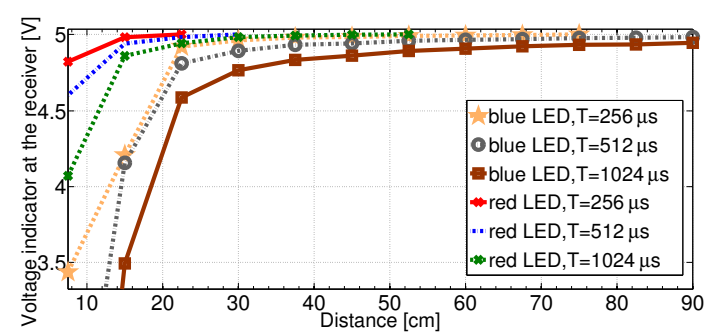

Fig. 8. Test of achievable range. The voltage is measured at the cathode pin of the LED receiver after a symbol period $T$, using 10-bit resolution. When the average voltage at the end of the period $T$ is below $5 \mathrm{~V}$, the transmitter detects light. The plot shows that the achievable distance is a function of $T$ and the LED itself and that the voltage follows an inverse square law decay. Distances of $90 \mathrm{~cm}$ and more can be obtained using blue LEDs and $T=[512,1024]$, while red LEDs can communicate only until $\approx 30 \mathrm{~cm}$.

\section{INSIGHTS ON LED-TO-LED PROTOCOL}

In this section, we give insights on our communication protocol and its implementation. We first focus on the MAC layer and show some example of data traffic using our prototype platform connected to a oscilloscope. We then consider the frame format at PHY layer.

MAC layer: Table I summarizes the main MAC parameters used in the implementation. The MAC_SlotTime duration is equal to 16 symbols, i.e., $(16 \cdot T)$. $\mathrm{RBT}_{\min }$ is equal to $2^{\text {MinExp }}=2^{4}$. MaxTry indicates the maximum number of attempts before a data is dropped. The ACK timeout indicates the time wherein the data transmitter waits for a valid frame after the end of data transmission. After each transition from busy channel (presence of energy in the Data symbols) to idle channel (absence of energy in the Data symbols), every station waits for an interframe space IFS before starting a new transmission or resuming the count down.

Fig. 9(a) displays a sequence of data and ACK frames, taken from the anode (TX) pins of two communicating stations. In order to facilitate the interpretation of the figure, in this example flicker elimination is activated only during data transmission and reception. Both stations transmit data in the slotted random access as described in Section III-C. In this example, station A (bottom) transmits a data frame after a backoff period time, which is received and replied with an ACK by station B (top). Then, after another idle time where both stations sense the channel activity, station B transmits the next data frame, and station A receives it and acknowledges it after the reception. Another message is then sent by station A, acknowledged by station B. In Fig. 9(b) we show an example using flicker compensation also inter-messages. While sensing the channel activity, both stations transmit symbol ONEs in the Energy symbols and messages can be successfully exchanged.

In Fig. 10, we show the data/ACK communication in a network of three stations. As above, to facilitate the interpretation of the figure, flicker elimination runs only during the message

TABLE I

MAC PARAMETERS

\begin{tabular}{|l|c||l|l|}
\hline MAC_SlotTime & 16 sym. & MaxTry & 11 \\
MinExp & 4 & ACKTimeout & 4 MAC_SlotTime \\
MaxExp & 13 & IFS & 2 MAC_SlotTime \\
\hline
\end{tabular}



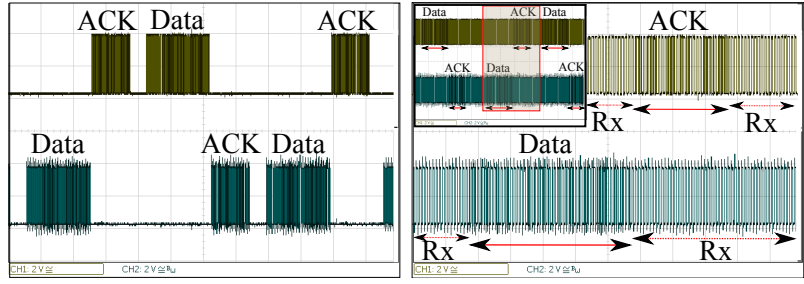

(a) Case with only intra-message (b) Case with full flickering eliminaflicker elimination.

Fig. 9. Measurement of two stations contending for the optical medium and sending data and acknowledgment (bottom: station A, top: station B).

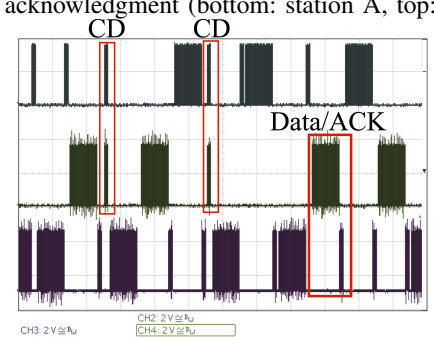

Fig. 10. CSMA/CD. Examples of collisions and successful message exchange using collision detection and ACKs. X-axis is time.

transmission and reception. The figure shows the voltage at the anode pin in each of the three stations. As in the example above, data/ACK communication can be observed. The plot also shows the collision detection mechanism, indicated as CD. Two stations stop their transmission as soon as they detect energy as explained in Section III-C1.

PHY layer: Fig. 11 illustrates the selected frame format, before coding the bits for anti-flickering elimination. On the physical layer, frames are constructed in the following way: a preamble which consists of sync sequence '1111 11111111 '. The sync pattern is followed by the so-called Special Frame Delimiter (SFD) to indicate the start of the message. We choose the SFD equal to '1101 1110'. Next is the message length field with the message length in byte, and the MAC header (MHR), with information such as the destination and source address. After the MHR, the data payload ( $P$ byte) is sent. We allow short messages of up to 160 byte to limit the system latency. A MAC footer containing the sixteen bit (Cyclic Redundancy Check, CRC) checksum over the MHR and data payload is appended. Bits are then encoded for antiflicker elimination. For example, the sync bit '11' will result in a sequence [ZERO ONE ONE ZERO] on the optical medium.

\section{CONCLUSION}

Today, visible light communication is often seen as yet another approach for in-home networking, device positioning, or a new way to access the internet. However, building a short-range free-space communication system by using LED transceivers instead of infrared or radio devices enables a wide variety of entertainment applications. In particular, these use

\begin{tabular}{|c|c|c|c|c|c|}
\hline 12 bit & 8 bit & 8 bit & 24 bit & $0-1280$ bit & 16 bit \\
\hline Sync & SFD & $\begin{array}{l}\text { Length } \text { MHR } \\
\text { Fig. 11. Frame format. }\end{array}$ & CRC \\
& & &
\end{tabular}

cases benefit from LEDs which can be used to emit as well as receive light. In this paper, we proposed and evaluated a complete LED-to-LED communication system including physical layer transmission and networking protocols, with focus on entertainment applications. We built and evaluated a prototype that enables the data exchange over short distances while avoiding radio emissions. Such benefits are for example the explicit visual feedback to the user when pointing a device, the system simplicity and the low-cost nature of the LEDbased systems. Our evaluation addressed technical challenges such as the elimination of flicker and introduced and evaluated a protocol for detecting collision. The results show that with one LED driven by a microcontroller, a system throughput of up to approximately 870 bit per second can be achieved in a network of LEDs on a selected light color, sufficient for entertaining interactive applications in the world of toys.

\section{REFERENCES}

[1] P. Dietz, W. Yerazunis, and D. Leigh, "Very Low-Cost Sensing and Communication Using Bidirectional LEDs," in TR2003-35, 2003.

[2] H. Elgala, R. Mesleh, and H. Haas, "Indoor optical wireless communication: Potential and state-of-the-art," IEEE Communications Magazine, Optical Communications Series, vol. 49, pp. 56-62, September 2011.

[3] S. Schmid, M. Gorlatova, D. Giustiniano, V. Vukadinovic, and S. Mangold, "Networking Smart Toys with Wireless ToyBridge and ToyTalk," in Poster Session, Infocom 2011, 2011.

[4] 802.15.7, "IEEE standard for local and metropolitan area networks, Part 15.7.," September 2011.

[5] N. O. Tippenhauer, D. Giustiniano, and S. Mangold, "Toys communicating with LEDs: Enabling Toy Cars Interaction," in Demo at Consumer Communications and Networking Conference, CCNC, IEEE, Jan. 2012.

[6] VLCC, "Visible Light Communications Consortium." http://www.vlcc. net, viewed 24-09-2012.

[7] Omega, "the Home Gigabit Access project." http://www.ict-omega.eu/, viewed 24-09-2012.

[8] F. Mims, "Sun photometer with light-emitting diodes as spectrally selective detectors," in Applied Optics, vol. 31, pp. 6965-6967, 1992.

[9] Y. Acharya, "Spectral and emission characteristics of LED and its application to LED-based sun-photometry," in Optics and Laser Technology, vol. 37, pp. 547-550, 2005.

[10] J. E. Farrell, "An analytical method for predicting perceived flicker," in Behaviour and Information Technology, vol. 5, pp. 349 - 358, 1986.

[11] S. Halpin, R. Bergeron, T. Blooming, R. Burch, L. Conrad, and T. Key, "Voltage and lamp flicker issues: Should the IEEE adopt the IEC approach,” IEEE Transactions on Power Delivery, pp. 1088-1097, 2003.

[12] C. J. Millett, D. R. Fish, and P. J. Thompson, "A survey of epilepsypatient perceptions of video-game material/electronic screens and other factors as seizure precipitants," Seizure, pp. 457 - 459, 1997.

[13] T. Yazaki, M. Hattori, and H. Tanaka, "Design and demonstration of $1 \mathrm{Gbit} / \mathrm{s}$ optical wireless interface for mobile phones," in GLOBECOM O7. IEEE, pp. $2226-2231$, nov. 2007.

[14] J. G. Proakis, Digital communications. McGraw-Hill Series in Electrical and Computer Engineering., 2001.

[15] S. Burns, A. Elsner, and M. Kreitz, "Analysis of nonlinearities in the flicker ERG," Optometry and Vision Science, pp. 95-105, 1992.

[16] D. Kelly, "Diffusion model of linear flicker responses," Journal of the Optical Society of America, pp. 1665-1670, 1969.

[17] Arduino, "Arduino Board Duemilanove." http://www.arduino.cc/en/ Main/ArduinoBoardDuemilanove, viewed 24-09-2012.

[18] R. Jain, D. Chiu, and W. Hawe, "A quantitative measure of fairness and discrimination for resource allocation in shared computer systems," Technical Report DEC-TR-301, September 1984.

[19] G. Bianchi, "Performance analysis of the IEEE 802.11 distributed coordination function," Selected Areas in Communications, IEEE Journal on, vol. 18, pp. 535 -547, March 2000.

[20] D. Kelly, "Indoor optical wireless communication: Potential and stateof-the-art," Journal of the Optical Society of America, 1971.

[21] M. Gorlatova, A. Wallwater, and G. Zussman, "Networking low-power energy harvesting devices: Measurements and algorithms," in INFOCOM, 2011 Proceedings IEEE, pp. 1602 -1610, april 2011. 